\title{
Frequência à creche e estado nutricional de pré-escolares: uma revisão sistemática
}

\author{
Day care centers attendance and preschool children's nutritional status: a systematic review
}

Alessandra da Silva Pereira' ${ }^{1}$, Haydée Serrão Lanzillotti ${ }^{2}$, Eliane de Abreu Soares ${ }^{3}$

\section{RESUMO}

Objetivo: Descrever e comparar estudos longitudinais que permitam inferir sobre a influência da creche no estado nutricional de crianças pré-escolares.

Fontes de dados: Revisão sistemática de trabalhos científicos publicados entre janeiro de 1990 e dezembro de 2008. Buscaram-se os estudos nas seguintes bases de dados: Lilacs, Scielo e PubMed. Realizou-se também pesquisa manual dos artigos referenciados. A busca ocorreu no período de março de 2008 a junho de 2009, e os descritores utilizados foram: "creche", "estado nutricional", "antropometria", "consumo alimentar”, "anemia” e "alimentação escolar”.

Síntese dos dados: Na primeira etapa do estudo, obtiveram-se 78 artigos, mas somente sete puderam ser incluídos. Os outros 71 não apresentaram dados para contribuir com o objetivo específico deste estudo. Entre os artigos pesquisados na literatura, existem poucos que permitem inferir sobre a influência que a creche pode ter em relação ao estado nutricional de pré-escolares. Contudo, estudos longitudinais têm mostrado a relação causal entre a presença frequente da criança na creche e a melhoria do estado nutricional.

Conclusões: Existe uma relação positiva entre a frequência da criança na creche e a melhoria do estado nutricional.

Palavras-chave: estado nutricional; pré-escolar; criança; antropometria; consumo de alimentos.

\section{ABTRACT}

Objective: To describe and compare longitudinal studies on how daycare centers influence preschoolers' nutritional status.

Data source: Systematic review of scientific papers published from January 1990 to December 2008. Studies were retrieved from the following databases: Lilacs, Scielo and PubMed. Manual search of papers was carried out as well. The review occurred between March 2008 and June 2009, and the searched terms were: "child day care center", "nutritional status", "anthropometry”, "food consumption”, "anemia" and "food program".

Data synthesis: In the first stage 78 papers were obtained, but only seven of them could be used. The other $71 \mathrm{did}$ not have data to contribute with the specific goal of this study. Among the papers gathered in the literature, very few of them allowed any inference about the influence of daycare centers on preschoolers' nutritional status. Nevertheless, some longitudinal studies showed a causal association between children's attendance to daycare centers and improvement of their nutritional status.

Conclusions: There is a positive relation between children's attendance to day care centers and improvement of their nutritional status.

Key-words: nutritional status; preschool, child; anthropometry; food consumption.
Instituição: Universidade do Estado do Rio de Janeiro (UERJ), Rio de Janeiro, RJ, Brasil

${ }^{1}$ Mestre em Alimentação, Nutrição e Saúde pela UERJ; Nutricionista da Secretaria de Educação da Prefeitura Municipal de Paraty, Rio de Janeiro, RJ, Brasil

2Doutora em Saúde Coletiva pelo Instituto de Medicina Social da UERJ; Professora Adjunta do Departamento de Nutrição Social do Instituto de Nutrição da UERJ, Rio de Janeiro, RJ, Brasil

${ }^{3}$ Doutora em Ciência dos Alimentos pela Universidade de São Paulo; Professora Associada do Departamento de Nutrição Básica e Experimental da UERJ, Rio de Janeiro, RJ, Brasil
Endereço para correspondência:

Alessandra da Silva Pereira

Rua Garibaldi, 163, apto. 702, Bloco 1 - Muda

CEP 20511-330 - Rio de Janeiro/RJ

E-mail: aspnutri@gmail.com

Conflitos de interesse: nada a declarar

Recebido em: 30/4/2009

Aprovado em: 26/9/2009 


\section{Introdução}

A saúde infantil, por ser um dos indicadores de saúde pública de um país e refletir as condições de vida de um local, desperta muito interesse no campo da pesquisa. Até alguns anos atrás, a desnutrição era o distúrbio nutricional mais frequentemente observado entre as crianças menores de cinco anos. Contudo, atualmente, além das carências nutricionais, estudos vêm apontando para o aumento da prevalência de sobrepeso em crianças, característico da transição nutricional ${ }^{(1-3)}$. Anemia ferropriva, hipovitaminose A e bócio também são carências nutricionais de alta prevalência no grupo materno-infantil ${ }^{(4,5)}$.

Os distúrbios nutricionais na infância estão relacionados com o desenvolvimento de doenças crônicas não-transmissíveis na vida adulta, sendo, portanto, a infância fase de extrema importância para ações de promoção da saúde ${ }^{(6,7)}$. O cenário detectado no Brasil pode ser consequência de modificações no padrão alimentar da população. Dados da Pesquisa de Orçamento Familiar - POF 2002/2003 ${ }^{(8)}$ apontam para o aumento no consumo de alimentos industrializados e a redução do consumo de cereais e leguminosas, frutas, legumes e verduras nas famílias brasileiras. A pré-escolaridade, por ser uma fase de intenso aprendizado, é fundamental para ações precoces de intervenção e monitoramento do estado nutricional ${ }^{(9)}$.

Diante do estilo contemporâneo da estrutura familiar, com maior participação da mulher no mercado de trabalho, houve considerável aumento na procura por creches, sobretudo as de tempo integral, para assistência de pré-escolares ${ }^{(10)}$. No Brasil, o acesso à creche de crianças na faixa etária de cinco a seis anos aumentou de 38\%, em 1991, para 72\%, em 2000(11). Segundo Bueno et al ${ }^{(12)}, 10$ a $15 \%$ de pré-escolares frequentam creches públicas nas grandes e médias cidades do Brasil.

Alguns estudos apontam que as creches podem ser consideradas como fator de proteção para o crescimento infantil, além de promoverem uma forma mais efetiva de intervenções coletivas em saúde ${ }^{(13-15)}$. Contudo, outros consideram a creche como fator de exposição, por exemplo, Vico e Laurenti ${ }^{(16)}$ encontraram associação entre o acesso à creche e o aumento da morbimortalidade em crianças, principalmente por doenças respiratórias. Gurgel et al ${ }^{(17)}$ observaram maior prevalência de infecções parasitárias intestinais em crianças frequentadoras de creches.

A relação entre frequência à creche e estado nutricional de pré-escolares vem despertando interesse no cenário atual, uma vez que, geralmente, a criança passa a maior parte do seu dia nessas instituições, em torno de nove horas por dia, o que faz com que as creches sejam responsáveis pelo fornecimento da maioria das refeições diárias. Além disso, essas instituições vêm deixando de ser somente "assistencialistas" para assumir um papel na formação das crianças, incluindo ações de promoção da saúde que interferem no estado nutricional dos pré-escolares.

A maioria dos estudos acerca da relação da creche com o estado nutricional de pré-escolares - que utilizam parâmetros antropométricos, dietéticos e/ou laboratoriais - é do tipo seccional, o que dificulta o estabelecimento de uma relação entre a frequência à instituição e uma possível melhora do estado nutricional. Para que se possa avaliar a efetividade da creche sobre o estado nutricional de pré-escolares, são necessários estudos do tipo longitudinal, que apresentem, no mínimo, duas observações ${ }^{(18)}$.

Diante das controvérsias sobre o estado nutricional de crianças assistidas por creches, optou-se pela realização de uma revisão sistemática cujo objetivo foi descrever e comparar estudos longitudinais que pudessem inferir sobre a influência da creche no estado nutricional de pré-escolares.

\section{Métodos}

O presente estudo é uma revisão sistemática acerca de trabalhos científicos que avaliaram a influência da creche no estado nutricional de pré-escolares, publicados entre janeiro de 1990 a dezembro de 2008, período escolhido devido à importância da década de 1990 para o crescimento da oferta de creches e educação infantil, consolidado pela Constituição Federal de 1988 e pela Lei de Diretrizes e Base de Educação Nacional de $1996^{(19)}$. A estratégia de pesquisa incluiu bases de dados eletrônicas - Lilacs, PubMed e Scielo - e busca in loco em periódicos e trabalhos científicos nas bibliotecas da Universidade do Estado do Rio de Janeiro e da Universidade Federal do Rio de Janeiro. O período de busca foi de março de 2008 a junho de 2009.

Para a realização da busca, utilizaram-se os descritores: "creche”, "estado nutricional”, "antropometria", "consumo alimentar", "anemia” e "alimentação escolar”. A pesquisa foi feita em português, inglês, francês e espanhol.

Os critérios de inclusão foram: estudos longitudinais publicados nos idiomas acima citados, com crianças entre 24 e 60 meses, frequentadoras de creche. Foram excluídos estudos transversais, estudos em outros idiomas e aqueles que contemplaram desfechos ou faixa etária diferentes dos desejados. Os estudos seccionais foram excluídos, tendo em vista o 
objetivo de reunir artigos que tivessem avaliado a efetividade da creche sobre o estado nutricional dos pré-escolares.

O processo de busca foi dividido em três etapas: na primeira, foram selecionados todos os artigos que atenderam os descritores; na segunda, os artigos selecionados foram filtrados, permanecendo somente aqueles que relacionavam a frequência à creche com o estado nutricional de pré-escolares; finalmente, na terceira etapa do processo, foram lidos e discutidos os artigos que atenderam a todos os critérios de inclusão.

\section{Resultados}

Na primeira etapa, utilizando-se os descritores mencionados, foram obtidos 78 artigos. Após leitura dos trabalhos selecionados, participaram da segunda etapa 50 estudos que relacionavam as creches com o estado nutricional dos pré-escolares. Nessa fase, excluíram-se nove artigos por terem sido realizados com crianças de faixa etária diferente da escolhida para o presente estudo, seis artigos por terem objetivo diferente deste trabalho e 21 por serem do tipo seccional. Excluíram-se dois artigos em chinês, um em japonês e um em alemão. Um trabalho foi excluído por estudar longitudinalmente pré-escolares sem vínculo obrigatório com uma creche. Dois trabalhos foram excluídos por estarem fora do período escolhido. Portanto, do total de artigos encontrados, sete foram identificados como compatíveis com o objetivo deste estudo. Dos artigos analisados, seis foram desenvolvidos no Brasil (cinco em São Paulo e um no Rio de Janeiro) e um no Egito. As características de cada um dos sete estudos estão detalhadas no Quadro 1.

Durante a busca bibliográfica que relacionasse a creche com o estado nutricional de pré-escolares, observou-se que a maior parte dos artigos encontrados foi do tipo transversal, ou seja, havia uma única avaliação do estado nutricional sem que se pudesse, portanto, avaliar a efetividade da frequência à instituição e mudanças no perfil nutricional dos pré-escolares. Apesar da importante contribuição dos estudos seccionais para os achados científicos, uma vez que muitos destes ${ }^{(20-23)}$ apresentam resultados que apontam para uma possível influência da frequência à creche no estado nutricional, optou-se por somente discutir os achados dos estudos longitudinais.

O número reduzido de estudos longitudinais encontrados possivelmente decorre das dificuldades operacionais características desse tipo de investigação, tais como: longo tempo de duração e alto custo, além de necessidade de equipe técnica especializada, entre outras. Contudo, mesmo em número reduzido quando comparados aos seccionais, a discussão dos artigos longitudinais acerca da hipótese levantada tem sua relevância pautada na necessidade de inferência científica sobre a efetividade dessas instituições para promover a saúde das crianças nelas assistidas.

O estado nutricional de crianças pode ser diretamente avaliado por meio de diferentes procedimentos, tais como: antropométrico, dietético, bioquímico e hematológico, contudo, considerando-se sempre que possível os diversos aspectos que indiretamente se relacionam, como os fatores sociodemográficos, psicossociais, econômicos e a história de saúde materno-infantil.

O perfil antropométrico é o mais amplamente utilizado, tanto para o diagnóstico, quanto para o monitoramento do estado nutricional, pois é de fácil operacionalização, baixo custo e não-invasivo. Os sete estudos longitudinais avaliados apresentaram parâmetros antropométricos. Dois deles obtiveram resultados do consumo dietético e três apresentaram parâmetros hematológicos.

A relação da creche com a evolução do perfil antropométrico foi evidenciada nesses sete estudos. Siviero et al ${ }^{(24)}$ acompanharam a evolução nutricional de 266 crianças de zero a seis anos e sete meses, consideradas em risco nutricional, antes e após intervenção realizada por meio de acompanhamento nutricional e atenção redobrada em creche pública de São Paulo, no período de abril a novembro de 1995. Os autores observaram melhora significativa nos índices altura/ idade, peso/altura e peso/idade, com sucesso na recuperação do estado nutricional das crianças desnutridas de 28,35 e $39 \%$, respectivamente.

Souza e Taddei ${ }^{(25)}$ avaliaram a relação entre intervenção nutricional (modificações dos cardápios oferecidos e fortificação do leite em pó) e o estado nutricional de 145 crianças com 11 meses a cinco anos e 11 meses, frequentadoras de uma creche comunitária em São Paulo. Os autores observaram redução significativa na prevalência de desnutrição, segundo a classificação de Gomez (1955) e a referência National Center for Health Statistics (NCHS) de 1977, não sendo a mesma redução observada com a classificação de Waterlow (1977). Os pesquisadores evidenciaram associação entre tempo de frequência na creche e modificação de categoria do estado nutricional. Crianças com maior exposição à creche apresentaram mobilidade do estado nutricional, ou seja, $11 \%$ migraram de baixo peso para eutrofia.

Taddei et a $l^{(26)}$ avaliaram a eficácia da creche na promoção de saúde em 168 crianças com risco de desnutrição, em estudo de monitoramento por nove meses, com avaliações periódicas do estado nutricional. Os pesquisadores observaram que, a partir do quarto mês, houve melhora no perfil 
Quadro 1 - Características dos estudos longitudinais que avaliaram o estado nutricional de crianças frequentadoras de creche

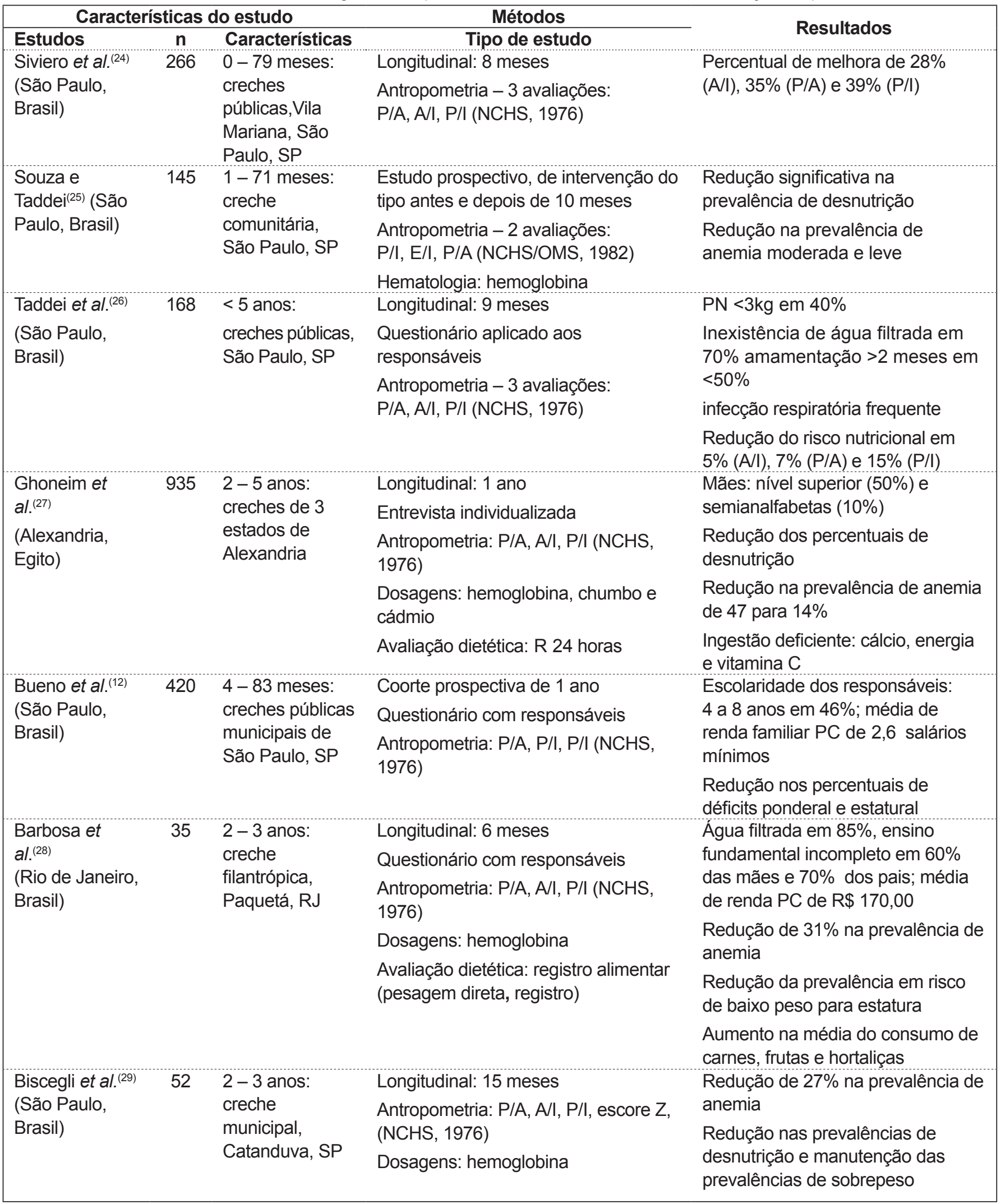

P/A: peso/altura; A/l: altura/idade; P/l: peso/idade; R: recordatório; PN: peso ao nascer; PC: per capita 
nutricional dos pré-escolares, com redução da desnutrição de 3; 15 e 45\%, respectivamente, para os índices peso/altura, peso/idade e altura/idade. Os autores destacam que a creche é efetiva para a melhora do estado nutricional de pré-escolares e provê oportunidade de educação para crianças menos privilegiadas, sendo, portanto, uma instituição de promoção social e da saúde.

Ghoneim $e t a^{(27)}$ realizaram um estudo longitudinal de intervenção durante um ano com 935 crianças de dois a cinco anos, em três creches de Alexandria (Egito). Os autores observaram redução no percentual de crianças com desnutrição de 4,6 para $1,5 \%$ e de 4,6 para $0,3 \%$ em meninos e meninas, respectivamente, segundo o índice peso/idade. No início do estudo, $5 \%$ dos meninos e $4,6 \%$ das meninas apresentavam déficit de estatura e, após um ano de intervenção, esses percentuais foram reduzidos para 3,5 e 2,3\%, respectivamente. Com relação ao índice peso/estatura, os pesquisadores observaram redução de desnutrição de $1,9 \%$ para zero entre os meninos e de $2,3 \%$ para $0,3 \%$ entre as meninas. Os autores relataram ter havido expressiva melhora do estado nutricional, considerando-se os três índices antropométricos avaliados.

Bueno $e t$ al ${ }^{(12)}$, após três avaliações de 420 crianças em intervalo de um ano, observaram redução de $1,7 \%$ no déficit peso/idade e de $3,1 \%$ no déficit altura/idade, entre a primeira e a terceira avaliação. Com relação ao índice peso/altura, os pesquisadores observaram um discreto aumento de $0,5 \%$. Apesar de os pesquisadores não terem estabelecido estatisticamente uma relação entre a frequência à creche e a evolução do estado nutricional, eles destacam que os resultados indicaram efeito positivo da creche sobre o crescimento infantil.

Barbosa et $a^{(28)}$ monitoraram o estado nutricional de 35 pré-escolares atendidos em uma creche filantrópica na Ilha de Paquetá, Rio de Janeiro. Em relação ao perfil antropométrico, as pesquisadoras observaram que, após seis meses de acompanhamento, houve melhora significativa do estado nutricional das crianças: a prevalência de risco nutricional para desnutrição segundo o índice peso/idade foi reduzida de $26 \%$ no primeiro momento para zero, no segundo. Apesar de não haver significância estatística, quando se avaliou o índice peso/estatura e estatura/idade, houve redução do risco nutricional de 26 para $8 \%$ e de 20 para $17 \%$, respectivamente. As pesquisadoras concluíram que a assistência oferecida na creche apresentou desempenho positivo no estado nutricional dos pré-escolares.

Biscegli et al ${ }^{(29)}$ realizaram estudo de coorte de 52 crianças de 32 a 78 meses, frequentadoras de uma creche no município de Catanduva, São Paulo, com o objetivo de avaliar o estado nutricional antes e após o tratamento para distúrbios nutricionais. Os autores, após 15 meses de intervenção, observaram redução significativa na prevalência de desnutrição (8 para $2 \%$ ), enquanto a prevalência de sobrepeso elevou-se de 7 para $8 \%$ - aumento não-significante. Porém, ao considerarem a redução na prevalência de desnutridos, a relação de obesos/desnutridos aumentou de $1 / 1$ para $4 / 1$, evidenciando mudança no padrão nutricional.

Ao avaliarem a influência da creche no consumo dietético de pré-escolares, Barbosa et al ${ }^{(28)}$ notaram diferença significante em relação ao consumo das porções dos diferentes grupos alimentares por 35 pré-escolares após seis meses de monitoramento, período em que as crianças participaram de diversas atividades de educação nutricional. Houve mudanças significativas quanto à ingestão de carnes, frutas e hortaliças: o consumo de porções do momento de ingresso na creche até seis meses depois aumentou de 2,4 para 2,9, de 1,0 para 2,2 e de 0,6 para 1,9 , respectivamente. O grupo de hortaliças, apesar de ter apresentado média de consumo aumentada entre os dois momentos, não atingiu a recomendação de três porções/dia ${ }^{(29)}$, diferente dos outros grupos de alimentos. Os autores calcularam ainda o Índice de Alimentação Saudável (HEI, do inglês Healthy Eating Index), uma medida para avaliar a qualidade da dieta, e verificaram que este se elevou de 77 para 93, concluindo que, após a entrada na creche, os pré-escolares apresentaram modificação no padrão alimentar, com consumo de maior variedade de alimentos e inclusão de alimentos saudáveis.

Ghoneim et al ${ }^{(27)}$ avaliaram o consumo alimentar de 935 pré-escolares de três creches e o comparam com as recomendações americanas (RDA: Recommended Daily Allowance). Os autores observaram que os nutrientes de menor consumo foram o cálcio (apenas 22\% das crianças consumiram quantidade $\geq 100 \%$ da RDA), energia (26\% consumiram quantidade $\geq 100 \%$ da RDA) e vitamina C ( $39 \%$ da RDA). Os nutrientes com maior consumo foram proteínas e vitaminas B1 e B2, que apresentaram percentual de crianças com consumo acima de $100 \%$ da RDA de 99 e $60 \%$, respectivamente. Os autores concluíram que programas de intervenção breves devem ser implantados em creches como estratégia de melhora do estado nutricional de crianças.

Com relação à avaliação hematológica em pré-escolares, o distúrbio mais amplamente avaliado foi a anemia, considerando-se principalmente os valores séricos de hemoglobina. Souza e Taddei ${ }^{(25)}$, em estudo de intervenção com fortificação do leite em pó com sais de ferro, oferecido a crianças 
frequentadoras de creche comunitária em São Paulo, observaram redução de 13 e $3 \%$, respectivamente, na prevalência de anemia moderada e leve após dez meses de intervenção.

Ghoneim et al ${ }^{(27)}$ avaliaram as concentrações séricas de hemoglobina de 148 crianças entre dois e cinco anos subpopulação das 935 crianças avaliadas em todo o estudo. Os autores definiram anemia quando a concentração sérica de hemoglobina foi inferior a $11 \mathrm{~g} / \mathrm{dL}$ e notaram redução na sua prevalência, com diminuição de 47 para 14\%, quando comparados os dois momentos, antes e após a intervenção.

Barbosa $e t$ a $l^{(28)}$ avaliaram a concentração de hemoglobina sérica de 35 pré-escolares de uma creche filantrópica, utilizando um hemoglobinômetro portátil. As autoras consideraram anêmicas as crianças com hemoglobina sérica menor que $12 \mathrm{~g} / \mathrm{dL}$. Após seis meses de monitoramento, a prevalência de anemia foi reduzida de 66 para $23 \%$.

Biscegli et $a^{(29)}$ avaliaram o estado de carência de ferro de 52 crianças de 32 a 78 meses antes e após 15 meses de tratamento com ferro, em estudo de coorte realizado entre setembro de 2005 e agosto de 2007, em creche de Catanduva. Os autores utilizaram para o diagnóstico de carência de ferro a avaliação da concentração sérica de hemoglobina, com pontos de corte de hemoglobina sérica menor que $11 \mathrm{~g} /$ $\mathrm{dL}$ para crianças de zero a 60 meses e menor que $12 \mathrm{~g} / \mathrm{dL}$ para crianças acima de 60 meses. $\mathrm{O}$ tratamento com ferro contou com dois esquemas de intervenção: 1) abordagem preventiva, com oferta de $1 \mathrm{mg} / \mathrm{kg} /$ dia por um período de três meses, realizada em 27 crianças, e 2) abordagem terapêutica de $3 \mathrm{mg} / \mathrm{kg} / \mathrm{dia}$ por três meses, utilizada em 25 crianças. Após a intervenção, os autores observaram redução significativa na prevalência de anemia (39 para 12\%).

Com relação às características socioeconômicas, os estudos avaliados apresentaram diferentes variáveis, não sendo possível compará-los. Contudo, ressaltam-se as variáveis mais relevantes de cada estudo. Bueno et al ${ }^{(12)}$ verificaram que a maioria das mães (79\%) estudou menos de oito anos e somente $1,2 \%$ contavam com ensino superior. A idade das mães variou entre 20 e 30 anos (50\%), sendo a média de idade observada de 29 anos. A média da renda familiar foi de 2,6 salários mínimos. Barbosa et al ${ }^{(28)}$ observaram que $60 \%$ das mães das crianças apresentavam escolaridade menor que oito anos e a média da renda per capita foi de $\mathrm{R} \$ 170,00$. Taddei et $a^{(26)}$ verificaram que $2 / 3$ das mães possuíam menos de cinco anos de estudo e $40 \%$ das crianças observadas nasceram com menos de $3000 \mathrm{~g}$. Os autores observaram ainda que $70 \%$ das famílias não possuíam água filtrada em casa e $15 \%$ das crianças apresentaram sintomas de diarreia e de infecções respiratórias no mês anterior ao estudo. Finalmente, Ghoneim et al ${ }^{(27)}$, na pesquisa realizada em Alexandria, no Egito, mostraram que mais de $50 \%$ das mães possuíam nível superior e somente $9,7 \%$ não sabiam ler e escrever. Esses achados diferem dos outros três estudos descritos acima devido principalmente à condição socioeconômica observada nas populações avaliadas. Ghoneim et al ${ }^{(27)}$ destacaram ainda que quase $70 \%$ das mães avaliadas estavam empregadas no momento da avaliação.

Os estudos apresentaram evidências positivas da relação entre frequência à creche e melhoria do estado nutricional de pré-escolares. Contudo, outros fatores relevantes ao estado nutricional de crianças, como os socioeconômicos, demográficos, genéticos e biológicos, nem sempre foram abordados e controlados pelos estudos, o que dificultou a análise comparativa.

Mesmo com os benefícios da creche para o estado nutricional de crianças, conforme observado nos estudos analisados, alguns autores ${ }^{(16,17)}$ apontam para um aumento na prevalência de morbidade em crianças frequentadoras de creches, principalmente infecções respiratórias e parasitárias, o que poderia indiretamente interferir no seu estado nutricional. Nesse contexto, ressalta-se a necessidade de mais estudos que avaliem a efetividade ou o impacto da creche no estado nutricional de pré-escolares, uma vez que tais instituições vêm se tornando mais presentes na vida das famílias contemporâneas e podem ser eficazes na promoção da saúde.

\section{Conclusão}

Apesar do número reduzido de estudos longitudinais que monitoraram o estado nutricional de crianças frequentadoras de creche, todos os trabalhos apresentados demonstraram relação positiva entre frequência à creche e segurança nutricional. 


\section{Referências bibliográficas}

1. Batista Filho M, Rissin A. Nutritional transition in Brazil: geographic and temporal trends. Cad Saúde Pública 2003;19:S181-91.

2. Silva GAP, Balaban G, Freitas MMV, Baracho JDS, Nascimento EMM. Overweight and obesity prevalence among preschool children of two private schools in Recife in the State of Pernambuco. Rev Bras Saude Mater Infant 2003;3:323-7.

3. Corso ACT, Botelho LJ, Zeni LA, Moreira EA. Overweight in children under 6 years of age in Florianópolis, SC, Brazil. Rev Nutr 2003;16:21-8.

4. Monteiro CA, Szarfarc SC, Mondine L. Secular trends in child anemia in S. Paulo city, Brazil (1984-1996). Rev Saúde Pública 2000;34:62-72.

5. Batista Filho M, Rissin A. Nutritional deficiencies: specific control measures by the health sector. Cad Saúde Pública 1993;9:130-5.

6. Pellanda LC, Echenique L, Barcellos LM, Maccari J, Borges FK, Zen BL. Ischemic heart disease: prevention should begin in childhood. J Pediatr (Rio J) 2002;78:91-6.

7. Pinheiro ARO, Freitas SFT, Corso ACT. An epidemiological approach to obesity. Rev Nutr 2004;17:523-33.

8. Brasil - IBGE [homepage on the Internet]. Pesquisa de Orçamentos Familiares - POF 2002-2003. Aquisição alimentar domiciliar per capita - Brasil e Grandes Regiões [cited 2009 Mar 15]. Available from: http://www.ibge.gov.br/home/ estatistica/populacao/condicaodevida/pof/2002aquisicao/default.shtm

9. Fagioli $D$, Nasser LA. Educação nutricional na infância e na adolescência: planejamento, intervenção, avaliação e dinâmica. São Paulo: RCN; 2006.

10. Barros AJD, Halpern R, Menegon OE. Creches públicas e privadas de Pelotas, RS: aderência à norma técnica. J Pediatr (Rio J) 1998;74:397-403.

11. Ministério Público do Estado de Santa Catarina [homepage on the Internet]. Educação: avanços e desafios [cited 2009 Mar 23]. Available from: http://www. mp.sc.gov.br/portal/site/portal/portal_detalhe.asp?campo $=2728$

12. Bueno MB, Marchioni DML, Fisberg RM. Evolução nutricional de crianças atendidas em creches públicas no Município de São Paulo, Brasil. Rev Panam Salud Publica 2003;14:165-70.

13. Corrêa AMS, Gonçalves NNS, Gonçalves A, Leite GPR, Padovani CR. Evolução da relação entre peso e altura e peso e idade em crianças de 3 meses a 6 anos assistidas em creche, Sorocaba (SP), Brasil. Rev Panam Salud Publica 1999;6:26-33.

14. Silva MV, Ometto AMH, Furtuoso MCO, Pipitone MAP, Sturion GL. Access to day-care centers and the nutritional status of Brazilian children: regional differences by age group and income class. Rev Nutr 2000;13:193-9.

15. Collet JP, Ducruet T, Floret D, Cogan-Collet J, Honneger D, Boissel JP. Day care attendance and risk of first infectious disease. Eur J Pediatr 1991;150:214-6.
16. Vico ESR, Laurenti R. Mortality among children enrolled in public day care centers in Brazil. Rev Saúde Pública 2004;38:38-44.

17. Gurgel RQ, Cardoso GS, Silva AM, Santos LN, Oliveira RCV. Children day care center: exposition or protection environment to intestinal parasites infestation in Aracaju, SE. Rev Soc Bras Med Trop 2005;38:267-9.

18. Victora CG, Habicht JP, Bryce J. Evidence-based public health: moving beyond randomized trials. Am J Public Health 2004;94:400-5.

19. Kuhlmann Jr M. Histórias da educação infantil brasileira. Rev Bras Educ 2000;14:5-18.

20. Temcharoen P, Laungintim P, Dhavidej C. The impact of Child Development Center on nutritional status and morbidity of early school age children. J Med Assoc Thai 1988; 71:10-14.

21. Poudel KC, Nakahara S, Okumura J, Wakai S. Day-care centre supplementary feeding effects on child nutrition in urban slum areas of Nepal. J Trop Pediatr 2004;50:116-9.

22. Real SI, Jaeger AS, Barón MA, Díaz N, Solano L, Velásquez E et al.. Estado nutricional en niños preescolares que asisten a un jardín de infancia público en Valencia, Venezuela. Arch latinoam nutr 2007;57:248-54

23. Castro TG, Novaes JF, Silva MR, Costa NMB, Franceschini SCC, Tinôco ALA et al. Characteristics of dietary intake, socioeconomic environment and nutritional status of preschoolers at public kindergartens. Rev Nutr 2005;18:321-30.

24. Siviero AA, Anti SM, Bandeira CR, Russeff MM, Fisberg M. Intervenção e orientação nutricional no acompanhamento de crianças desnutridas em creches de São Paulo. Rev Paul Pediatr 1997;15:7-13.

25. Souza PC, Taddei JA. Efeito da freqüência à creche nas condições de saúde e nutrição de pré-escolares residentes em favelas da periferia de São Paulo, 1996. Rev Paul Pediatr 1998;16:143-50.

26. Taddei JA, Cannon MJ, Warner L, Souza P, Vitalle S, Palma D et al. Nutritional gains of underprivileged children attending a day care center in S. Paulo City, Brazil: a nine month follow-up study. Rev Bras Epidemiol 2000;3:29-37.

27. Ghoneim EH, Hassan MH, Amine EK. An intervention programme for improving the nutritional status of children aged 2-5 years in Alexandria. East Mediterr Health J 2004;10:828-43.

28. Barbosa RMS, Salles-Costa R, Soares EA. Estado nutricional de crianças atendidas em uma creche filantrópica: estudo longitudinal de 6 meses. Alim Nutr 2007;18:215-21.

29. Biscegli TS, Corrêa CEC, Romera J, Candido AB. Nutritional status and iron deficiency among children enrolled in a day care center before and after 15 months of nutritional management. Rev Paul Pediatr 2008;26:124-9. 